\title{
Formulação geometricamente exata de estruturas de cabos de aço com a configuração parabólica
}

\author{
Rodrigo Sernizon Costa ${ }^{1 *}$; Armando Cesar Campos Lavall ${ }^{2 *}$; Renata Gomes \\ Lanna da Silva ${ }^{3}$; Ricardo Hallal Fakury ${ }^{4}$ \\ $1^{*}$ Escola Politécnica; Universidade Federal da Bahia; Rua Prof. Aristides Novis, \\ 02, Federação - Salvador, CEP 40210-630, Bahia, Brasil; \\ rodrigo.sernizon@ufba.br \\ $2^{*}$ Escola de Engenharia; Universidade Federal de Minas Gerais; Av. Antônio \\ Carlos, 6627, Pampulha - Belo Horizonte, CEP 31270-901, Minas Gerais, Brasil; \\ lavall@dees.ufmg.br \\ ${ }^{3}$ Centro Federal de Educação Tecnológica de Minas Gerais; Av. Amazonas, 5.253, Nova \\ Suíça, Belo Horizonte, MG, Brasil. CEP: 30.421-169, Minas Gerais, Brasil; \\ rglanna.silva@gmail.com \\ ${ }^{4}$ Escola de Engenharia; Universidade Federal de Minas Gerais; Av. Antônio \\ Carlos, 6627, Pampulha - Belo Horizonte, CEP 31270-901, Minas Gerais, Brasil; \\ fakury@dees.ufmg.br
}

\section{Geometrically exact formulation of steel cables structures with parabolic configuration}

\section{Resumo}

Este trabalho tem por objetivo apresentar uma formulação geometricamente exata para análise estática de estruturas de cabos de aço suspensos, via método dos elementos finitos, considerando a configuração inicial parabólica do cabo. Para isso, foram consideradas ambas as não linearidades, geométrica e do material, para o elemento de cabo. Na formulação numérica tridimensional foi considerada uma rigorosa formulação Lagrangiana atualizada, que utiliza a técnica corrotacional para a dedução consistente da matriz de rigidez tangente do elemento de cabo. Por fim, são apresentados exemplos da literatura cujos resultados são comparados com aqueles obtidos pelo software desenvolvido com base na formulação apresentada, visando demonstrar a consistência e eficiência da formulação.

Palavra-chave: Formulação geometricamente exata, Estruturas de cabos de aço suspensos, Cabo parabólico, Análise elastoplástica.

\begin{abstract}
The objective of this work is to present a geometrically exact formulation for the static analysis of suspended steel cables structures, using the finite element method, considering the initial parabolic configuration of the cable. For this, both geometric and material nonlinearities, were considered for the cable element. In the three-dimensional numerical formulation an accurate updated Lagrangian formulation was considered, which uses the corrotational technique for the consistent deduction of the tangent stiffness matrix of the cable element. Finally, examples of the literature are presented, whose results are compared with those obtained by the software developed based on the presented formulation, aiming to demonstrate the consistency and efficiency of the formulation.
\end{abstract}

Keywords: Geometrically exact formulation, Suspended steel cables structures, Parabolic cable, Elastoplastic analysis.

* autor correspondente 


\section{Introdução}

Cabos são elementos estruturais de grande utilização na engenharia, como por exemplo, em linhas de transmissão de energia elétrica, torres estaiadas, teleféricos, coberturas e pontes penseis e estaiadas. O seu comportamento é relativamente complexo quando comparados aos demais elementos estruturais.

Os cabos apresentam comportamento geométrico altamente não linear, como também afirmam Kim et al. (2015) e Abad et al. (2013), são extremamente flexíveis e capazes de resistir a forças normais de tração. Teoricamente, considera-se que as forças normais de compressão, as forças cortantes, os momentos fletores e os momentos de torção não são resistidos por um cabo ideal.

O comportamento não linear geométrico do cabo é obtido utilizando uma análise que considera o seu equilíbrio na sua posição deslocada, através de uma análise geometricamente exata. Além disso, os cabos possuem um comportamento não linear material devido a própria não linearidade da sua lei constitutiva. Dessa forma, o estudo do cabo, além de envolver o desenvolvimento das relações não lineares entre forças e deslocamentos, exige a complexa tarefa de se obter a configuração geométrica inicial das estruturas de cabos.

Portanto, este trabalho tem como objetivo desenvolver uma formulação numérica geometricamente exata via método dos elementos finitos, visando à realização de uma análise estática de estruturas de cabos suspensos parabólicos. Nessa análise consideram-se as não linearidades, geométrica e material, e a obtenção da configuração inicial do cabo admitindo-a, inicialmente, parabólica.

\section{Formulação analítica para os cabos suspensos}

Inicialmente, será apresentada uma formulação analítica para os cabos suspensos, considerando-se a hipótese que o cabo seja perfeitamente flexível, dessa forma, não oferecendo resistência à flexão e, consequentemente, a força normal de tração atuante será sempre tangente à sua geometria, sendo, portanto, variável ao longo de seu comprimento. Será considerada também a hipótese de que o cabo seja inextensível, isto é, o cabo apresenta o mesmo comprimento antes e depois da aplicação da carga. Portanto, uma vez aplicadas as ações externas, a geometria deformada permanece inalterada e o cabo, ou cada segmento do cabo, pode ser tratado como corpo rígido. 
Essa formulação analítica é adotada apenas para a determinação, de forma aproximada, da configuração inicial do cabo, que dependendo do carregamento externo, o cabo pode assumir diferentes configurações.

Os tipos de carregamentos podem ser feitos por meio de cargas concentradas, cargas uniformemente distribuídas ao longo do vão do cabo e carga uniformemente distribuída ao longo do comprimento do cabo (peso próprio). Nessas duas últimas situações de carregamentos obtêm-se as configurações iniciais de equilíbrio, parabólica e em forma de catenária, respectivamente, onde a catenária é utilizada por Abad et al. (2013). Quando o carregamento distribuído ao longo do vão é muito maior do que o peso próprio do cabo, o efeito da catenária pode ser desprezado na análise.

Um estudo analítico dos cabos suspensos considerando-se as hipóteses citadas, conforme fazem Irvine (1975) e (1981), Leonard (1988), Beer et al. (2006), Hibbeler (2011) e Costa (2014), será apresentado a seguir, visando à implementação computacional para obtenção da configuração inicial parabólica de equilíbrio do cabo.

\subsection{Cabo parabólico}

Um cabo suspenso por dois pontos $A$ e $B$, é ilustrado na Figura 1. São identificados nessa figura o vão do cabo $L$, a distância do ponto $A$ ao vértice da curva do cabo $x_{v}$, os ângulos de inclinação do cabo $\theta_{A}$ e $\theta_{B}$ nos pontos $A$ e $B$, respectivamente, a flecha máxima no vértice da curva $f_{v}$ e o desnível $h$ entre os pontos de fixação do cabo.

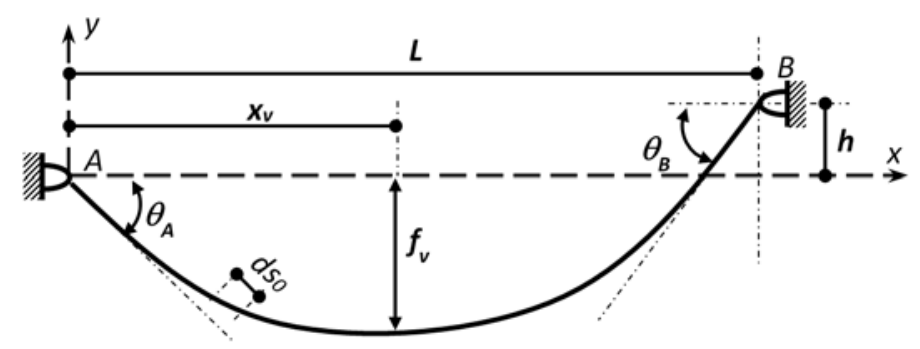

Figura 1 - Configuração inicial do cabo suspenso

Admitindo-se que o cabo está submetido a um carregamento uniformemente distribuído, $w(x)$, ao logo do seu vão $L$, a Figura 2 ilustra o diagrama de corpo livre de um elemento infinitesimal do cabo com comprimento $d S_{0}$ na sua posição de equilíbrio. Também são ilustradas as forças horizontais $H_{0}$ e $H_{0}+d H_{0}$, as forças verticais $V_{0}$ e $V_{0}+d V_{0}$ nas extremidades $j$ e $k$, bem como o ângulo de inclinação $\theta$ do elemento de cabo. 


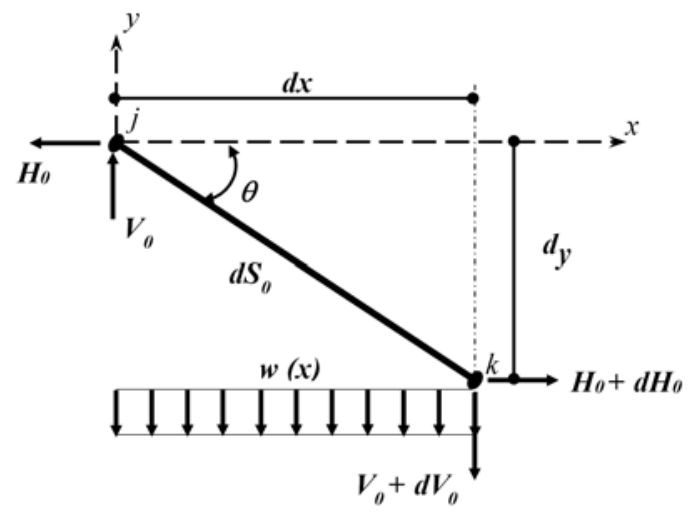

Figura 2 - Elemento infinitesimal de cabo sujeito a um carregamento uniformemente distribuído

Aplicando-se as equações de equilíbrio no elemento, desprezando-se os termos de ordem superior, chega-se às seguintes expressões:

$$
\begin{aligned}
& \Sigma H=0 \rightarrow d H_{0}=0 \\
& \sum V=0 \rightarrow d V_{0}=-w d x \\
& \sum M_{k}=0 \rightarrow \frac{d y}{d x}=y^{\prime}=\frac{V_{0}}{H_{0}}
\end{aligned}
$$

Sendo $d H_{0}=0$, pode-se concluir que a componente horizontal $H_{0}$ de força no cabo é constante. Derivando-se a Eq. (3) e com auxílio da Eq. (2), tem-se a Eq. (4), que representa a equação diferencial do elemento de cabo na sua posição de equilíbrio.

$$
y^{\prime \prime}=-\frac{w}{H_{0}}
$$

Integrando-se a Eq. (4) tem-se a tangente à curva do cabo ou rotação $\theta$, dada pela expressão:

$$
y^{\prime}=-\frac{w}{H_{0}} x+C_{1}
$$

Integrando-se a Eq. (5), chega-se na equação da curva do cabo, que é representada por uma parábola, dada pela Eq. (6).

$$
y=-\frac{w}{2 H_{0}} x^{2}+C_{1} x+C_{2}
$$

onde as constantes $C_{1}$ e $C_{2}$ dependem das condições de contorno da estrutura.

Aplicando-se as condições de contorno da estrutura ilustrada na Figura 1, onde se tem para $x=0 \rightarrow y_{A}^{\prime}=\tan \left(\theta_{A}\right)$ e para $x=0 \rightarrow y_{A}=0$, determinam-se as constantes $C_{1}$ e $C_{2}$, dadas respectivamente, por:

$$
\begin{aligned}
& C_{1}=\tan \left(\theta_{A}\right) \\
& C_{2}=0
\end{aligned}
$$


Substituindo-se os valores das constantes $C_{1}$ e $C_{2}$ nas Eqs. (5) e (6), encontram-se a equação da tangente à curva do cabo, Eq. (9) e a equação da configuração de equilíbrio do cabo, dada pela equação da parábola, Eq. (10).

$$
\begin{aligned}
& y^{\prime}=-\frac{w}{H_{0}} x+\tan \left(\theta_{A}\right) \\
& y=-\frac{w}{2 H_{0}} x^{2}+\tan \left(\theta_{A}\right) x
\end{aligned}
$$

Para se calcular a componente horizontal $\boldsymbol{H}_{0}$ da força de tração no cabo é necessário aplicar as seguintes condições de contorno: para $x=L \rightarrow y_{B}=h$, que substituindo na Eq. (10) tem-se:

$$
H_{0}=\frac{w L^{2}}{2\left[L \tan \left(\theta_{A}\right)-h\right]}
$$

Com auxílio da Figura 2 , o comprimento do cabo, $S_{0}$, pode ser obtido através da seguinte expressão:

$$
d S_{0}=\sqrt{(d x)^{2}+(d y)^{2}}=\left(\sqrt{1+\left(y^{\prime}\right)^{2}}\right) d x
$$

Substituindo-se a Eq. (9) na Eq. (12) e integrando-a, obtém-se a equação do comprimento do cabo, Eq. (13).

$S_{0}=-\frac{H_{0}}{2 w}\left\{\left[-\frac{w L}{H_{0}}+\tan \left(\theta_{A}\right)\right] \sqrt{\left[-\frac{w L}{H_{0}}+\tan \left(\theta_{A}\right)\right]^{2}+1}-\tan \left(\theta_{A}\right) \sec \left(\theta_{A}\right)+\sinh ^{-1}\left[-\frac{w L}{H_{0}}+\right.\right.$ $\left.\left.\tan \left(\theta_{A}\right)\right]-\sinh ^{-1}\left[\tan \left(\theta_{A}\right)\right]\right\}$

Quando não se necessita de grande exatidão, pode-se utilizar a aproximação $\sqrt{1+a} \cong$ $1+\frac{a}{2}$, se $a$ é um valor muito pequeno. Então, a Eq. (13) fica:

$$
S_{0} \cong L\left\{1+\frac{w^{2} L^{2}}{6 H_{0}^{2}}-\frac{\tan \left(\theta_{A}\right)}{2}\left[\frac{w L}{H_{0}}-\tan \left(\theta_{A}\right)\right]\right\}
$$

A força de tração no cabo, $T$, tendo-se em vista as suas componentes $H_{0}$ e $V_{0}$ da Figura 2, onde $H_{0}$ é constante, pode ser escrita por:

$$
\mathrm{T}=\frac{H_{0}}{\cos \theta}
$$

Sabendo-se que $\cos \theta=\frac{d x}{d s_{0}}$, com o auxílio das Eqs. (12) e (9), obtém-se à força de tração no cabo que varia continuamente em intensidade e direção ao longo de toda a sua extensão.

$$
T=H_{0} \sqrt{1+\left[-\frac{w}{H_{0}} x+\tan \left(\theta_{A}\right)\right]^{2}}
$$


A Eq. (16) mostra que a força de tração no cabo $(T)$ é função, além do carregamento uniforme distribuído $(w)$, dos parâmetros $H_{0}$ e $\tan \left(\theta_{A}\right)$.

Voltando-se à Figura 1, supondo-se que não se conheça o ângulo $\theta_{A}$, mas se conheça a abscissa do vértice $\left(x_{v}\right)$, substitui-se na Eq. (9) a condição de contorno em que para $x=x_{v} \rightarrow y^{\prime}=0$, e na Eq. (10) a condição em que para $x=L \rightarrow y_{B}=h$, obtendo-se:

$$
\begin{aligned}
& \tan \left(\theta_{A}\right)=\frac{w}{H_{0}} x_{v} \\
& H_{0}=-\frac{w L}{2 h}\left(L-2 x_{v}\right)
\end{aligned}
$$

Levando-se a Eq. (18) na Eq. (17) encontra-se a expressão da tangente de $\theta_{A}$ em função dos dados do problema, dada por:

$$
\tan \left(\theta_{A}\right)=-\frac{2 h x_{v}}{L\left(L-2 x_{v}\right)}
$$

Analogamente, voltando-se à Figura 1, supondo-se que a variável conhecida seja a flecha do vértice $\left(f_{v}\right)$ e substituindo-se na Eq. (10) a condição de contorno em que para $x=x_{v} \rightarrow y=f_{v}$, e com o auxílio da Eq. (17), obtém-se:

$$
f_{v}=\frac{H_{0}}{2 w} \tan ^{2}\left(\theta_{A}\right)
$$

Levando-se a Eq. (11) na Eq. (20) obtém-se:

$$
\tan \left(\theta_{A}\right)=\frac{2 f_{v}}{L}\left(1+\sqrt{1-\frac{h}{f_{v}}}\right)
$$

Levando-se a Eq. (21) na Eq. (11) encontra-se uma nova expressão da força horizontal, $H_{0}$, em função dos dados do problema, dada por:

$$
H_{0}=\frac{w L^{2}}{2 f_{v}\left(1+\sqrt{1-\frac{h}{f_{v}}}\right)^{2}}
$$

\section{Formulação numérica para os cabos suspensos}

O Método dos Elementos Finitos (MEF) é utilizado para análise não linear com intuito de estudar as estruturas de cabos considerando o sistema tridimensional. A teoria é desenvolvida através de uma rigorosa formulação Lagrangiana atualizada que utiliza a técnica corrotacional para a dedução consistente das matrizes de rigidez dos elementos de cabo espacial.

A formulação apresentada, baseada, principalmente, nos trabalhos de Pimenta (1989), Lavall (1996), Fábio (2000) e Costa (2014), pretende ser a mais geral possível, permitindo que os nós sofram grandes deslocamentos e os elementos de cabos sofram grandes alongamentos e, além disso, esses elementos podem ser constituídos de material 
elastoplástico e considerar tanto o comportamento não linear geométrico (NLG) quanto não linear do material (NLM).

\subsection{Deformações e tensões}

Em mecânica dos sólidos para que uma análise teórica seja consistente, as medidas de deformações e tensões devem ser conjugadas e objetivas, conforme Bathe $(1982,1996)$ e Pai e Nayfeh (1994). As deformações de engenharia formam um par conjugado com as tensões de engenharia ou nominais. Ao se adotar o sistema local de coordenadas corrotacionais no desenvolvimento da formulação, pode-se garantir que as deformações e tensões de engenharia são, também, pares de medidas de deformações e tensões objetivas e, dessa forma, as deformações e tensões de engenharia serão utilizadas como referência neste trabalho, sendo designadas, respectivamente, por:

$$
\begin{gathered}
\varepsilon=\frac{l_{c}-l_{r}}{l_{r}} \\
\sigma_{N}=\frac{N}{A_{r}}
\end{gathered}
$$

onde $I_{r}$ é o comprimento do elemento na configuração de referência, $I_{c}$ é o comprimento do elemento na configuração corrigida; $N$ é a força normal e $A_{r}$ é a área da seção transversal do elemento.

\subsection{Sistema de coordenadas e graus de liberdade}

Fundamentando-se o desenvolvimento teórico em uma formulação Lagrangiana, o sistema de referência global adotado neste trabalho é o sistema de coordenadas cartesiano $(X, Y, Z)$, conforme ilustra a Figura 3. Para o sistema local adotou-se o sistema de coordenadas corrotacionais $(x, y, z)$, diferente do sistema global de referência. Esse sistema está ligado ao elemento, no qual os deslocamentos generalizados são medidos em relação a uma configuração deformada. Trata-se, portanto, de um sistema de referência móvel que acompanha a estrutura deformada.

Considerando-se as coordenadas corrotacionais de referência $\left(x_{r}, y_{r}, z_{r}\right)$ com origem no centro do elemento de cabo, os ângulos $\varphi_{r z}, \varphi_{r x}$ e $\varphi_{r y}$ são formados entre o eixo do elemento e os eixos os globais $(X, Y, Z)$, respectivamente. Após um carregamento qualquer o elemento passa para uma nova posição com coordenadas corrotacionais corrigidas $\left(x_{c}, y_{c}, z_{c}\right)$, formando-se novos ângulos $\varphi_{c z}, \varphi_{c x}$ e $\varphi_{c y}$ entre o eixo do elemento de cabo e os eixos de referência global $(X, Y, Z)$. Um elemento de cabo apresenta somente deformação longitudinal, assim, o grau de liberdade natural ou corrotacional $\left(q_{\alpha}=q_{1}\right)$ associado a essa deformação pode ser definido por: 


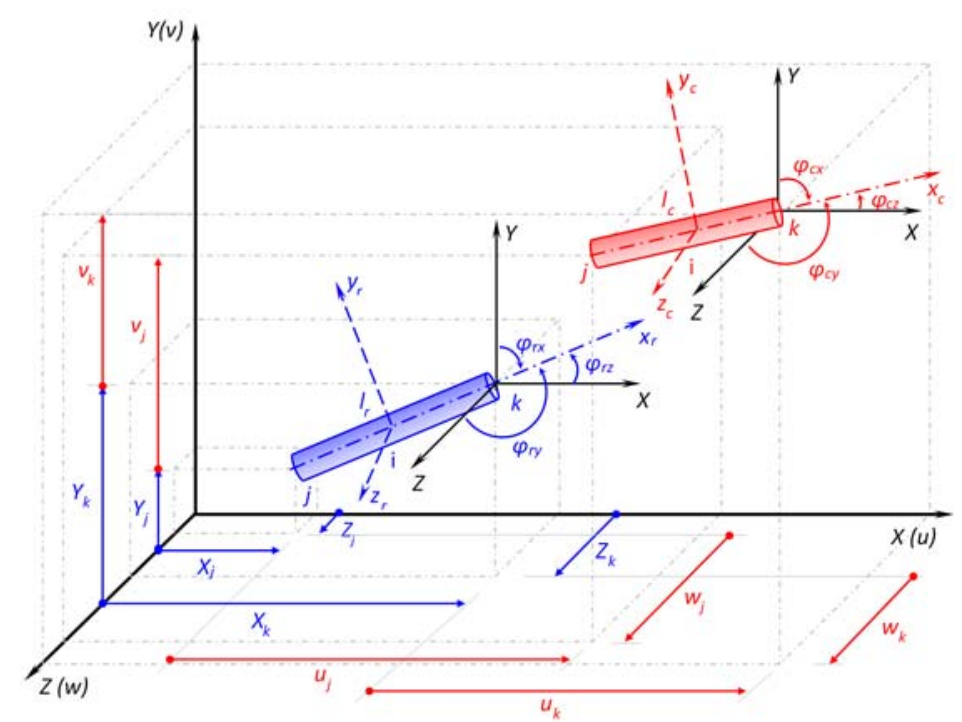

Figura 3 - Elemento de cabo nas configurações de referência e corrigida

$$
q_{1}=l_{c}-l_{r}
$$

Os graus de liberdade cartesianos $p_{n}(\mathrm{n}=1$ a 6 ) podem ser definidos pelo vetor de deslocamentos nodais do elemento de extremidade $j$ e $k$, dado por:

$$
\boldsymbol{p}_{n}^{T}=\left\{\begin{array}{llllll}
u_{j} & v_{j} & w_{j} & u_{k} & v_{k} & w_{k}
\end{array}\right\}
$$

Considerando a Fig.3, o grau de liberdade em coordenadas corrotacionais $q_{\alpha}$, e os graus de liberdade em coordenadas globais cartesianas $p_{n}$, podem ser relacionados conforme as expressões a seguir:

$$
\begin{aligned}
& l_{r}=\sqrt{\left(\Delta X^{2}+\Delta Y^{2}+\Delta Z^{2}\right)} \\
& l_{r}=\sqrt{\left[(\Delta X+\Delta u)^{2}+(\Delta Y+\Delta v)^{2}+(\Delta Z+\Delta w)^{2}\right]}
\end{aligned}
$$

sendo, $\Delta X=X_{k}-X_{j} ; \Delta Y=Y_{k}-Y_{j} ; \Delta Z=Z_{k}-Z_{j} ; \Delta u=u_{k}-u_{j}=p_{4}-p_{1} ; \Delta v=v_{k}-v_{j}=$ $p_{5}-p_{2} ; \Delta w=w_{k}-w_{j}=p_{6}-p_{3}$, onde $X_{j}, X_{k}, Y_{j}, Y_{k}, Z_{j}$ e $Z_{k}$ são as coordenadas dos elementos na configuração global de referência.

Substituindo os valores das Eqs. (27) e (28) na Eq. (25) e derivando-se as coordenadas locais corrotacionais, $\boldsymbol{q}_{\alpha}$, em relação às coordenadas globais cartesianas, $\boldsymbol{p}_{n}$, conforme Costa (2014), obtém-se a matriz $\boldsymbol{B}_{1 \times 6}$, ou seja, $\partial q_{\alpha} / \partial p_{n}$, que pode ser escrita também na forma indicial por $\boldsymbol{q}_{\alpha, n}$.

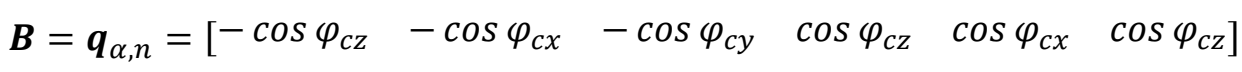

onde $\boldsymbol{B}$ é uma matriz de mudança de coordenadas que relaciona as taxas de deslocamentos nas coordenadas locais corrotacionais com as taxas de deslocamentos nas coordenadas globais cartesianas. 
Derivadas de segunda ordem de $\boldsymbol{q}_{\alpha}$ em relação a $\boldsymbol{p}_{n}$, ou seja, $\partial^{2} q_{\alpha} / \partial p_{n} \partial p_{m},(\alpha=1$, $n=m=1$ a 6 ) ou $\boldsymbol{q}_{\alpha, n m}$, são também necessárias e podem ser reunidas em uma matriz simétrica $\boldsymbol{G}_{\alpha(6 \times 6) \text {, dada por: }}$

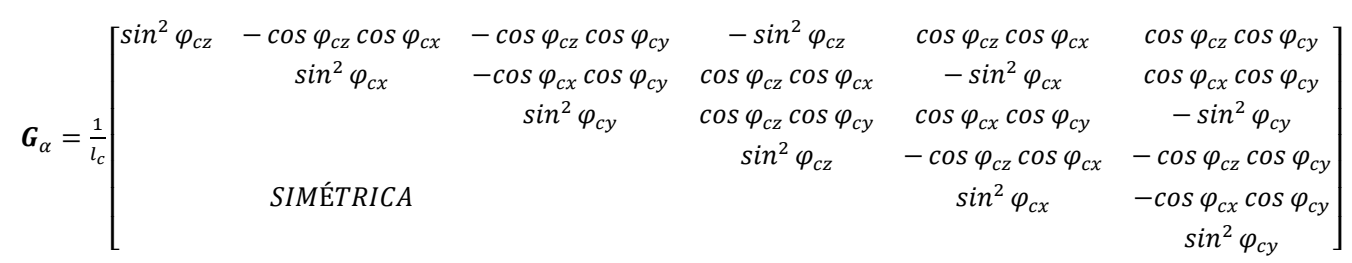

\subsection{Equilíbrio do elemento}

Observa-se das Eqs. (23), (27) e (28) que o campo de deformação pode ser descrito em função dos graus de liberdade naturais, dado por:

$$
\varepsilon=f\left[q_{\alpha}\left(p_{n}\right)\right]
$$

Conhecido esse campo de deformação, obtém-se o equilíbrio do elemento por meio do Princípio dos Trabalhos Virtuais (PTV). O trabalho virtual interno de um elemento resulta em:

$$
\delta w_{\text {int }}=\int_{V_{r}} \sigma \delta \varepsilon d V_{r}
$$

sendo $\sigma$ a tensão normal de um elemento, $\delta \varepsilon$ a deformação virtual desse elemento, $d V_{r}$ o elemento de volume na configuração de referência.

A deformação virtual é obtida utilizando-se a regra da cadeia e pode ser escrita por:

$$
\delta \varepsilon=\varepsilon_{, \alpha} q_{\alpha, n} \delta p_{n}
$$

onde $\delta p_{n}$ o vetor dos deslocamentos nodais virtuais do elemento.

As forças nodais internas $P_{n}$, oriundas das ações externas, são definidas de maneira que:

$$
\delta w_{\text {int }}=P_{n} \delta p_{n}
$$

Igualando-se a Eq. (32) e a Eq. (34) com auxílio da Eq. (33), obtém-se a equação de equilíbrio dado por:

$$
P_{n}=\int_{V_{r}} \sigma \varepsilon_{, \alpha} q_{\alpha, n} d V_{r}
$$

Definindo-se os esforços internos nas coordenadas corrotacionais ou naturais, $Q_{\alpha}$, por:

$$
Q_{\alpha}=\int_{V_{r}} \sigma \varepsilon_{, \alpha} d V_{r}
$$

A equação de equilíbrio do elemento, escrita em notação indicial, é dada por:

$$
P_{i}=Q_{\alpha} q_{\alpha, n}
$$

Escrevendo a Eq. (37) na forma matricial tem-se:

$$
P=B^{T} Q
$$

onde $\boldsymbol{P}$ são as forças nodais internas nas coordenadas cartesianas e $\boldsymbol{Q}$ são os esforços internos nas coordenadas corrotacionais. 


\subsection{Matriz de rigidez tangente do elemento}

A matriz de rigidez tangente do elemento, $k_{t}$, nas coordenadas cartesianas, definida pela derivada dos esforços nodais internos $P_{n}$, Eq. (37), em relação aos deslocamentos nodais do elemento $p_{m}$, é dada por:

$$
\boldsymbol{k}_{\boldsymbol{t}}=\frac{\partial P_{n}}{\partial p_{m}}
$$

A Eq. (39) pode ser escrita em notação matricial, como:

$$
\dot{\boldsymbol{P}}=\boldsymbol{k}_{\boldsymbol{t}} \dot{\boldsymbol{p}}
$$

Realizando as devidas operações matemáticas obtém-se a matriz de rigidez tangente do elemento dada pela seguinte expressão:

$$
k_{n m}=q_{\alpha, n} D_{\alpha \beta} q_{\beta, m}+q_{\alpha, n} H_{\alpha \beta} q_{\beta, m}+Q_{\alpha} q_{\alpha, n m}
$$

Observa-se na Eq. (41) a parcela constitutiva representada por $q_{\alpha, n} D_{\alpha \beta} q_{\beta, m}$, e as parcelas geométricas representadas por $\left(q_{\alpha, n} H_{\alpha \beta} q_{\beta, m}\right)$ e $\left(Q_{\alpha} q_{\alpha, n m}\right)$ que consideram os conhecidos efeitos " $P$ - $\delta$ " e " $P-\Delta$ ", respectivamente.

A forma matricial da Eq. (41) é dada por:

$$
k_{t}=B^{T} D B+B^{T} H B+Q_{\alpha} G_{\alpha}
$$

\section{Aspectos da implementação computacional}

O programa desenvolvido em Costa (2014) foi adaptado de Lavall (1996) e escrito na linguagem FORTRAN 90 utilizando a plataforma Microsoft Visual Studio 2008. Este programa, nomeado como Advanced STRuctural Analysis System (ASTRAS), permite realizar análises estáticas não lineares, geométrica e material, de estruturas.

Visando à solução de problemas não lineares de estruturas, foi utilizado o Método de Newton Raphson que considera incrementos de cargas com iterações de equilíbrio realizadas dentro de cada passo. Neste trabalho foram implementados os critérios de convergência em deslocamento e forças, simultaneamente, podendo-se também verificar a convergência somente no deslocamento ou na força, sendo o fator de tolerância igual a $0,1 \%$, em quaisquer dos casos citados.

\subsection{Implementação da configuração inicial de equilíbrio do cabo suspenso}

Para geração dos nós e elementos das estruturas de cabos é necessária a implementação da configuração inicial de equilíbrio do cabo. Para atingir o objetivo desse estudo foi implementada no programa ASTRAS, a configuração inicial de equilíbrio do cabo sujeito a um carregamento uniformemente distribuído (cabo parábola).

A configuração inicial de equilíbrio do cabo é dada pela equação da parábola, Eq. (10), que depende do cálculo da componente horizontal, $H_{0}$, da força de tração do cabo para 
geração dos nós e elementos da estrutura. No estudo desenvolvido na seção 2.1 , a componente horizontal $\left(H_{0}\right)$ do cabo parábola é dada pela Eq. (11) ou Eq (18) ou Eq. (22).

\subsection{Análise incremental das tensões e deformações no comportamento elastoplástico de estruturas de cabos de aço suspensos}

Conforme Costa (2014), neste trabalho propõe-se um diagrama tensão $(\sigma)$ versus deformação $(\varepsilon)$ que, dividido em seis trechos lineares, torna possível a simulação numérica do comportamento elastoplástico das estruturas de cabos de aço, conforme ilustrado na Figura 4.

Considerando que os elementos de cabo não resistem à compressão, o trecho I corresponde às tensões e deformações nulas. O trecho II é definido pela lei de Hooke, atribuindo ao material a rigidez elástica $(E)$. Os trechos de III a VI, simulam o comportamento elastoplástico, com módulo de rigidez tangente $\left(E_{t i}\right)$ e parâmetro de encruamento $\left(H_{i}\right)$, com $i$ variando de 1 a 4.

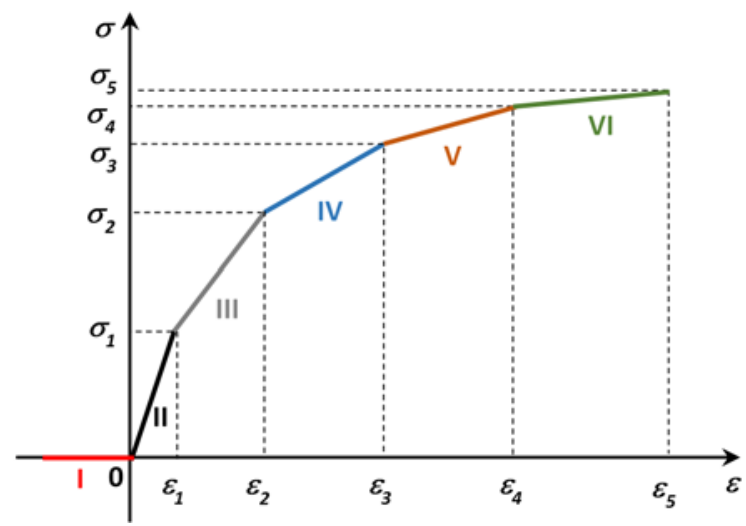

Figura 4 - Diagrama multilinear tensão versus deformação

\section{Aplicação em exemplos numéricos}

Nessa seção são apresentados exemplos numéricos com o objetivo de avaliar a eficiência da formulação, bem como a precisão dos resultados obtidos pelo programa ASTRAS, quando comparados com resultados teóricos da literatura. O primeiro exemplo considera apenas a não linearidade geométrica para um cabo suspenso sujeito à cargas concentradas e distribuída ao longo do vão. O segundo exemplo considera as análises não lineares, geométrica e material, de uma estrutura formada por três cabos.

\subsection{Análise não linear geométrica}

O cabo da Figura 5 está sujeito às forças concentradas $P_{C}=200 \mathrm{kN}$ e $P_{D}=100 \mathrm{kN}$ aplicadas às distâncias $L_{1}=4.000 \mathrm{~cm}$ e $L_{2}=8.000 \mathrm{~cm}$ do apoio $A$, respectivamente, além do carregamento uniformemente distribuído $q(x)=0,05 \mathrm{kN} / \mathrm{cm}$ ao longo do vão $L=10.000 \mathrm{~cm}$. 


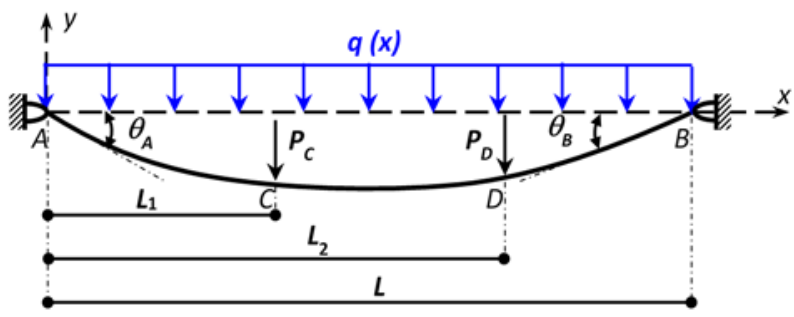

Figura 5 - Cabo suspenso sujeito à carga uniformemente distribuída e à forças concentradas

Para a análise numérica o cabo $A B$ foi dividido em 20 elementos contendo um total de 21 nós, sendo que os trechos $A C, C D$ e $D B$ contêm 8,8 e 4 elementos, respectivamente. Admitiu-se uma configuração inicial de equilíbrio obtida pela equação da parábola, Eq. (10), com uma flecha inicial $f=1.005 \mathrm{~cm}$ e peso próprio $w=0,001 \mathrm{kN} / \mathrm{cm}$. O cabo tem seção transversal circular com diâmetro $d=3,57 \mathrm{~cm}$ e módulo de elasticidade $E=165.000 \mathrm{MPa}$.

A Tabela 1 apresenta os resultados do comprimento $\left(S_{0}\right)$, da flecha máxima $\left(f_{\text {máx }}\right)$, dos ângulos $\theta_{A}$ e $\theta_{B}$, da tração máxima no cabo ( $\left.T_{\text {máx }}\right)$ e das reações de apoio $H_{A}, V_{A}, H_{B}$ e $V_{B}$, visando à comparação entre o processo analítico de Süssekind (1987) e o programa ASTRAS.

Os valores numéricos foram obtidos aplicando-se 100 incrementos de carga do peso próprio do cabo para o posicionamento inicial de equilíbrio e 100 incrementos referentes às forças concentradas e ao carregamento uniformemente distribuído. A convergência em deslocamentos e forças, para uma tolerância de 0,1\%, necessitou apenas de três iterações. O processamento computacional foi de cinquenta segundos utilizando-se um computador DELL XPS com processador Intel core i5, CPU de 1,80 GHz, memória RAM de 8GB e 256 GB de SSD.

Tabela 1 - Resultados analíticos (Süssekind, 1987) e numéricos (ASTRAS)

\begin{tabular}{cccc}
\hline Variáveis & $\begin{array}{c}\text { Süssekind } \\
(\mathbf{1 9 8 7})^{[\mathrm{a}]}\end{array}$ & ASTRAS $^{[\mathrm{b}]}$ & $\begin{array}{c}\text { Erro relativo } \\
([\mathrm{a}]-[\mathrm{b}]) /[\mathrm{a}](\%)\end{array}$ \\
\hline \hline$S_{0}(\mathrm{~cm})$ & $10.328,00$ & $10.327,45$ & 0,0053 \\
$f_{\text {máx }}(\mathrm{cm})$ & $1.160,00$ & $1.158,41$ & 0,1371 \\
$\theta_{A}$ (graus) & 21,31 & 20,74 & 2,6748 \\
$\theta_{B}$ (graus) & 22,29 & 21,70 & 2,6469 \\
$T_{\text {máx }}(\mathrm{kN})$ & $1.080,80$ & $1.087,15$ & $-0,5875$ \\
$H_{A}(\mathrm{kN})$ & $1.000,00$ & $1.010,10$ & $-1,0100$ \\
$H_{B}(\mathrm{kN})$ & $1.000,00$ & $1.010,10$ & $-1,0100$ \\
$V_{A}(\mathrm{kN})$ & 390,00 & 395,25 & $-1,3461$ \\
$V_{B}(\mathrm{kN})$ & 410,00 & 414,75 & $-1,1585$ \\
\hline
\end{tabular}


Observando-se a Tabela 1, verifica-se que os resultados analíticos e numéricos têm boa correlação. Nota-se que os resultados referentes à geometria da estrutura $\left(S_{0}, f_{\text {máx }}, \theta_{A} \mathrm{e}\right.$ $\theta_{B}$ ), obtidos da formulação proposta (programa ASTRAS), são menores do que os resultados obtidos da formulção analítica (Sussekind). Isto ocorre porque a parcela da matriz de rigidez global da estrutura de cabo, obtida através da formulação proposta, referente à matriz de rigidez geométrica, contribui para aumentar a rigidez da estrutura, uma vez que a força normal atuante no cabo é de tração. Isto forma o sistema estrutural mais rígido, levando a menores deslocamentos da estrutura. Consequentemente, isto implica em um aumento nos valores das forças atuantes $\left(T_{\text {máx }}, H_{A}, H_{B}, V_{A}, V_{B}\right)$. Apesar dos deslocamentos envolvidos neste exemplo serem pequenos, os resultados alcançados mostram claramente a contribuição da matriz de rigidez geométrica na análise, o que não ocorre na formulação analítica, validando a análise não linear geométrica proposta.

A Figura 6 ilustra as configurações inicial e final do cabo após o carregamento. Observase claramente a configuração inicial parabólica do cabo e a configuração final onde os segmentos $A C, C D$ e $D B$ não são retos, conforme se considera no processo analítico de resolução de estruturas de cabo.

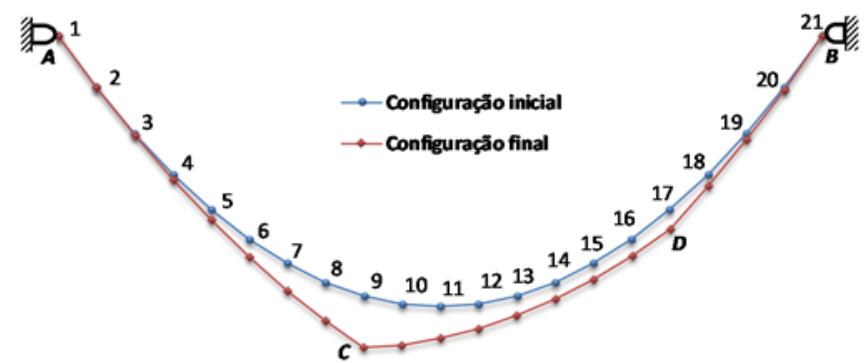

Figura 6 - Configurações inicial e final da estrutura de cabo

\subsection{Análise não linear material e geométrica}

Este exemplo tem como objetivo avaliar os efeitos das não linearidades, geométrica e material, da estrutura formada por três cabos, conforme mostra a Figura 7. Duas leis constitutivas para o material dos cabos de aço serão estudadas, conforme as Figuras 8 e 10, para a avaliação da análise incremental de tensões e deformações apresentadas na seção 4.2 .

Considere nessa estrutura que os cabos $\overline{\mathrm{AD}}, \overline{\mathrm{BD}}$ e $\overline{\mathrm{CD}}$ têm os mesmos módulos de elasticidade longitudinal do aço $E=20.000 \mathrm{kN} / \mathrm{cm}^{2}$, a mesma tensão limite de escoamento $\sigma_{y}=34,50 \mathrm{kN} / \mathrm{cm}^{2}$ e a mesma seção transversal circular com diâmetro $d=4,0 \mathrm{~cm}$, sendo fornecido o comprimento $L=200 \mathrm{~cm}$ e a carga aplicada no ponto $D$, $P=1.050 \mathrm{kN}$. 


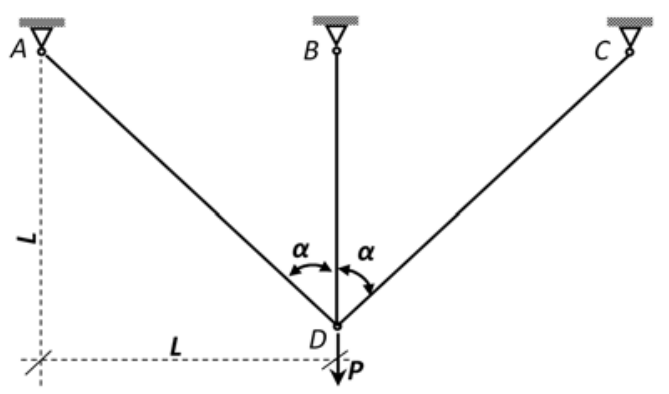

Figura 7 - Estrutura hiperestática plana de 3 cabos

Através dos conceitos da mecânica dos sólidos aplicando-se o Método da Forças, temse, na fase elástica:

$$
\begin{aligned}
& F_{A D}=F_{C D}=\frac{P_{i} \cos ^{2}(\alpha)}{1+2 \cos ^{3}(\alpha)} \\
& F_{B D}=\frac{P_{i}}{1+2 \cos ^{3}(\alpha)} \\
& \delta_{D}=\frac{\left(P_{i}-F_{B D}\right) L}{2 E A \cos ^{3}(\alpha)}=\frac{F_{B D} L}{E A} \\
& P_{y}=\sigma_{y} A\left[1+2 \cos ^{3}(\alpha)\right]
\end{aligned}
$$

onde, $P_{i}$ é a carga no incremento $i ; \alpha$ é o ângulo entre os elementos $\overline{\mathrm{AD}}$ e $\overline{\mathrm{BD}}$, e $\overline{\mathrm{CD}}$ e $\overline{\mathrm{BD}}$, que neste caso vale $\alpha=45^{\circ} ; F_{A D}$ e $F_{C D}$ são os esforços que atuam nos cabos $\overline{\mathrm{AD}}$ e $\overline{\mathrm{CD}}$, respectivamente; $F_{B D}$ é o esforço que atua no cabo $\overline{\mathrm{BD}} ; \delta_{D}$ é o deslocamento vertical no ponto $D, A$ é a área da seção transversal e $P_{y}$ é a carga que provoca o escoamento do elemento de cabo $\overline{\mathrm{BD}}$.

Após o escoamento do elemento de cabo $\overline{\mathrm{BD}}$, os esforços nos elementos e o deslocamento no ponto $D$ serão calculados pelas seguintes expressões:

$$
\begin{aligned}
& F_{A D}=F_{C D}=\frac{P_{i}-\sigma_{y} A}{2 \cos (\alpha)} \\
& F_{B D}=\sigma_{y} A \\
& \delta_{D}=\frac{\left(P_{i}-P_{y}\right) L}{2 E A \cos ^{3}(\alpha)}+\frac{F_{B D} L}{E A}
\end{aligned}
$$

A estrutura entrará em colapso quando se atingir a carga última, $P_{u}$, dada por:

$$
P_{u}=\sigma_{y} A[1+2 \cos (\alpha)]
$$

\section{A) Lei Constitutiva 1}

A lei constitutiva 1, conforme a diagrama de tensões versus deformações da Figura 8, considera o comportamento elastoplástico perfeito, sendo compreendido pelo trecho I, no qual consideram-se tensões nulas quando as deformações são de compressão, pelo trecho II que é elástico e pelo trecho III plástico, sendo $\sigma_{y}$ e $\varepsilon_{y}$ a resistência e a deformação no início do escoamento, respectivamente. 
Aplicando-se cargas incrementais, $P_{i}$, em 11 incrementos conforme os percentuais acumulados do fator de carga, $f_{c}$, indicados na Tabela 2, até o carregamento máximo $P=1.050 \mathrm{kN}$, utilizaram-se as Eqs. (43) a (50) para obtenção dos resultados analíticos também mostrados na Tabela 2. Na Tabela 3 são fornecidos os resultados obtidos pelo programa ASTRAS.

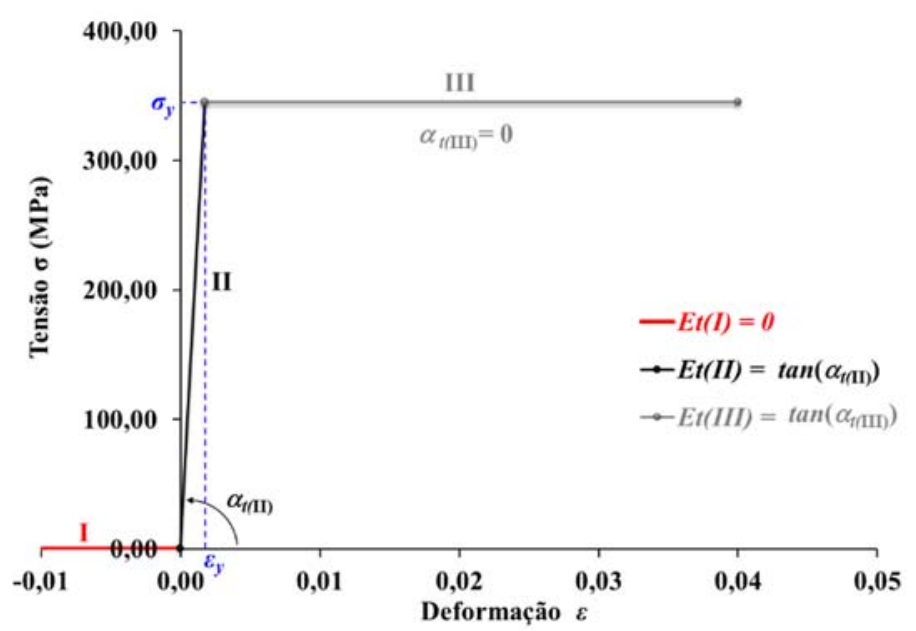

Figura 8 - Comportamento elastoplástico perfeito (lei constitutiva 1)

Tabela 2 - Resultados analíticos da análise referente à lei constitutiva 1

\begin{tabular}{|c|c|c|c|c|c|c|c|c|}
\hline $\begin{array}{c}P_{y} \\
\text { (kN) }\end{array}$ & $\begin{array}{c}P_{u} \\
(k N)\end{array}$ & $i$ & $\begin{array}{l}\Sigma f_{c} \\
(\%)\end{array}$ & $\begin{array}{c}\Sigma P_{i} \\
(\mathrm{kN})\end{array}$ & $\begin{array}{c}F_{A D} \\
(\mathbf{k N})\end{array}$ & $\begin{array}{c}F_{B D} \\
(k N)\end{array}$ & $\begin{array}{c}\boldsymbol{F}_{C D} \\
(\mathbf{k N})\end{array}$ & $\begin{array}{c}\delta_{D} \\
(\mathrm{~cm})\end{array}$ \\
\hline \multirow{11}{*}{740,10} & \multirow{11}{*}{$1.046,66$} & 1 & 20,00 & 210,00 & 61,508 & 123,015 & 61,508 & 0,098 \\
\hline & & 2 & 40,00 & 420,00 & 123,015 & 246,030 & 123,015 & 0,196 \\
\hline & & 3 & 60,00 & 630,00 & 184,523 & 369,045 & 184,523 & 0,294 \\
\hline & & 4 & 70,49 & 740,10 & 216,768 & 433,536 & 216,768 & 0,345 \\
\hline & & 5 & 75,00 & 787,50 & 250,288 & 433,540 & 250,288 & 0,398 \\
\hline & & 6 & 80,00 & 840,00 & 287,411 & 433,540 & 287,411 & 0,457 \\
\hline & & 7 & 85,00 & 892,50 & 324,534 & 433,540 & 324,534 & 0,517 \\
\hline & & 8 & 90,00 & 945,00 & 361,657 & 433,540 & 361,657 & 0,576 \\
\hline & & 9 & 95,00 & 997,50 & 398,780 & 433,540 & 398,780 & 0,635 \\
\hline & & 10 & 99,68 & $1.046,65$ & 433,530 & 433,540 & 433,530 & 0,690 \\
\hline & & 11 & 100,00 & $1.050,00$ & \multicolumn{4}{|c|}{ Colapso da estrutura } \\
\hline
\end{tabular}

Observa-se nas Tabelas 2 e 3 que os esforços nos elementos de cabo, assim como os valores dos deslocamentos, mostram uma excelente precisão entre os resultados analíticos e numéricos. Até $70,49 \%$ do carregamento $\left(P_{4}=740,10 \mathrm{kN}\right)$ todos os 
elementos de cabo trabalham em regime elástico contribuindo para a rigidez do sistema.

A partir desta carga, pode-se verificar pela força $F_{B D}$ que o elemento $\overline{\mathrm{BD}}$ escoa, permanecendo com esforço constante, $\left(F_{B D}=433,54 \mathrm{kN}\right)$, deixando de contribuir para a rigidez da estrutura. Neste momento apenas os elementos $\overline{\mathrm{AD}}$ e $\overline{\mathrm{CD}}$ resistem aos esforços adicionais e a rigidez do sistema diminui, conforme indica a mudança de inclinação da curva da Figura 9.

Tabela 3 - Resultados numéricos da análise referente à lei constitutiva 1

\begin{tabular}{ccccccccc}
\hline $\begin{array}{c}\boldsymbol{P}_{\boldsymbol{y}} \\
(\mathbf{k N})\end{array}$ & $\begin{array}{c}\boldsymbol{P}_{u} \\
(\mathbf{k N})\end{array}$ & $\boldsymbol{i}$ & $\begin{array}{c}\boldsymbol{\Sigma} \boldsymbol{f}_{\boldsymbol{c}} \\
\mathbf{( \% )}\end{array}$ & $\begin{array}{c}\boldsymbol{\Sigma} \boldsymbol{P}_{\boldsymbol{i}} \\
\mathbf{( k N )}\end{array}$ & $\begin{array}{c}\boldsymbol{F}_{A D} \\
(\mathbf{k N})\end{array}$ & $\begin{array}{c}\boldsymbol{F}_{B D} \\
(\mathbf{k N})\end{array}$ & $\begin{array}{c}\boldsymbol{F}_{C D} \\
(\mathbf{k N})\end{array}$ & $\begin{array}{c}\boldsymbol{\delta}_{D} \\
(\mathbf{c m})\end{array}$ \\
\hline & & 20,00 & 210,00 & 61,506 & 122,996 & 61,506 & 0,098 \\
& & 2 & 40,00 & 420,00 & 123,008 & 245,956 & 123,008 & 0,196 \\
& & 3 & 60,00 & 630,00 & 184,506 & 368,877 & 184,506 & 0,294 \\
& & 4 & 70,49 & 740,10 & 216,745 & 433,304 & 215,254 & 0,345 \\
& & 5 & 75,00 & 787,50 & 250,039 & 433,540 & 250,039 & 0,398 \\
& $1.050,00$ & 6 & 80,00 & 840,00 & 287,084 & 433,540 & 287,084 & 0,457 \\
& & 7 & 85,00 & 892,50 & 324,117 & 433,540 & 324,117 & 0,516 \\
& & 8 & 90,00 & 945,00 & 361,140 & 433,540 & 361,14 & 0,574 \\
& 9 & 95,00 & 997,50 & 398,151 & 433,540 & 398,151 & 0,633 \\
& & 10 & 99,68 & $1.046,65$ & 432,792 & 433,540 & 432,792 & 0,688 \\
& 11 & 100,00 & $1.050,00$ & 433,540 & 433,540 & 433,540 & 2,199 \\
\hline
\end{tabular}

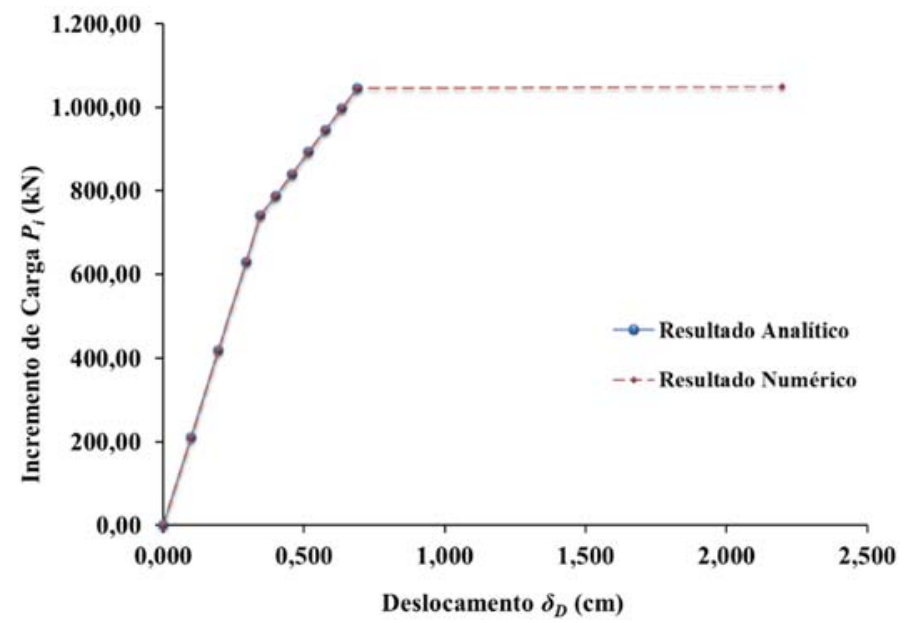

Figura 9 - Curva carga versus deslocamento vertical em $D\left(\delta_{D}\right)$ (lei constitutiva 1 )

No décimo primeiro incremento $\left(P_{u}=1.050,00 \mathrm{kN}\right)$ pode-se verificar que ocorre 0 colapso da estrutura, sendo que no processo numérico é possível determinar as forças de tração nos elementos $\overline{\mathrm{AD}}$ e $\overline{\mathrm{CD}}$, bem como o deslocamento vertical final no ponto $D$ $\left(\delta_{D}=2,199 \mathrm{~cm}\right)$. Dessa forma, observa-se que ao se utilizar a formulação teórica 
desenvolvida neste trabalho é possível estudar o equilíbrio da estrutura em sua nova posição, devido à contribuição da parcela da rigidez geométrica na análise (não linearidade geométrica).

Para este caso, foram necessárias no máximo cinco iterações para a convergência da solução e o tempo de processamento computacional total foi de dois segundos, utilizando-se um computador DELL XPS com processador Intel core i5, CPU de 1,80 GHz, memória RAM de 8 GB e 256 GB de SSD.

\section{B) Lei Constitutiva 2}

Para estrutura da Figura 7, será adotada agora a lei constitutiva 2 com comportamento elastoplástico conforme diagrama tensão $(\sigma)$ versus deformação $(\varepsilon)$ apresentada na Figura 10 , onde $\sigma_{y}$ é a resistência ao escoamento.

Os valores limites das tensões $(\sigma)$ e deformações $(\varepsilon)$ que definem cada trecho do comportamento do material e os seus respectivos módulos tangentes $\left(E_{t}\right)$ estão apresentados na Tabela 4.

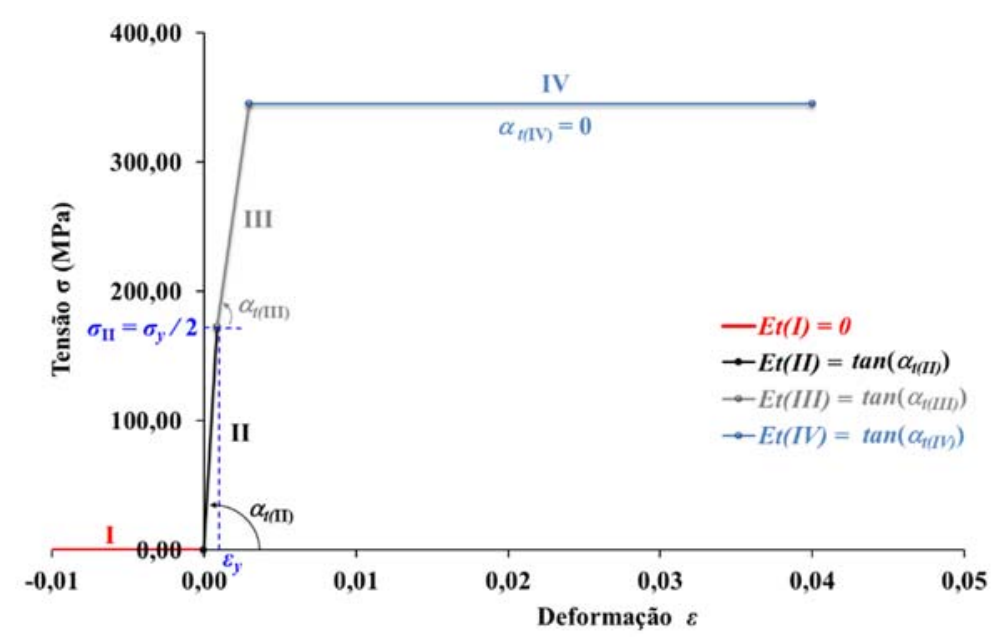

Figura 10 - Comportamento elastoplástico - lei constitutiva 2

Tabela 4 - Tensões, deformações e módulo tangente da lei constitutiva 2

\begin{tabular}{ccccc}
\hline Trecho & Comportamento & $\begin{array}{c}\sigma \\
(\mathrm{MPa})\end{array}$ & $\boldsymbol{\varepsilon}$ & $\begin{array}{c}\boldsymbol{E}_{\boldsymbol{t}} \\
\text { (MPa) }\end{array}$ \\
\hline \hline I & - & 0,00 & 0,00 & 0,00 \\
II & Elástico & 172,50 & 0,0008625 & 200.000 \\
III & Elastoplástico & 345,00 & 0,0029661 & 82.000 \\
IV & Plástico & 345,00 & 0,04 & 0,00 \\
\hline
\end{tabular}

A Tabela 5 fornece os resultados obtidos pelo programa ASTRAS utilizando a lei constitutiva 2, sendo $f_{c}$ o fator de carga, $\varepsilon_{t 1}$ a deformação total dos cabos $\overline{\mathrm{AD}}$ e $\overline{\mathrm{CD}}$ e $\varepsilon_{t 2}$ 
a deformação total do cabo $\overline{\mathrm{BD}}$. A Figura 11 ilustra a curva da carga versus deslocamentos verticais no ponto $D$, referente às análises segundo a lei constitutiva 2 em comparação com a lei constitutiva 1.

Tabela 5 - Resultados numéricos da análise referentes a lei constitutiva 2

\begin{tabular}{ccccccccc}
\hline $\boldsymbol{i}$ & $\begin{array}{c}\boldsymbol{\Sigma} \boldsymbol{f}_{\boldsymbol{c}} \\
(\%)\end{array}$ & $\begin{array}{c}\boldsymbol{\Sigma} \boldsymbol{P}_{\boldsymbol{i}} \\
\mathbf{( k N )}\end{array}$ & $\begin{array}{c}\boldsymbol{F}_{A D} \\
(\mathbf{k N})\end{array}$ & $\begin{array}{c}\boldsymbol{F}_{B D} \\
\mathbf{( k N )}\end{array}$ & $\begin{array}{c}\boldsymbol{F}_{C D} \\
\mathbf{( k N )}\end{array}$ & $\begin{array}{c}\boldsymbol{\delta}_{D} \\
(\mathbf{c m})\end{array}$ & $\boldsymbol{\varepsilon}_{t 1}$ & $\boldsymbol{\varepsilon}_{t 2}$ \\
\hline \hline 1 & 20,00 & 210,00 & 61,506 & 122,996 & 61,506 & 0,098 & 0,000245 & 0,000489 \\
2 & 35,00 & 367,50 & 107,633 & 215,219 & 107,633 & 0,171 & 0,000428 & 0,000856 \\
3 & 60,00 & 630,00 & 221,923 & 315,867 & 221,923 & 0,365 & 0,000913 & 0,001824 \\
4 & 70,48 & 740,09 & 254,144 & 380,239 & 254,144 & 0,490 & 0,001225 & 0,002449 \\
5 & 75,00 & 787,50 & 268,018 & 407,951 & 268,018 & 0,544 & 0,001360 & 0,002718 \\
6 & 80,00 & 840,00 & 286,969 & 433,540 & 286,969 & 0,617 & 0,001544 & 0,003085 \\
7 & 85,00 & 892,50 & 323,920 & 433,540 & 323,920 & 0,760 & 0,001902 & 0,003801 \\
8 & 90,00 & 945,00 & 360,845 & 433,540 & 360,845 & 0,903 & 0,002261 & 0,004516 \\
9 & 95,00 & 997,50 & 397,744 & 433,540 & 397,744 & 1,046 & 0,002619 & 0,005231 \\
10 & 99,68 & $1.046,65$ & 432,265 & 433,540 & 432,265 & 1,180 & 0,002954 & 0,005899 \\
11 & 100,00 & $1.050,00$ & 433,540 & 433,540 & 433,540 & 2,199 & 0,005511 & 0,010993 \\
\hline
\end{tabular}

Analisando a Tabela 5 e a Figura 11 podem-se distinguir três etapas do comportamento da estrutura em sua análise considerando a lei constitutiva 2. A primeira etapa corresponde ao início do processo incremental até o segundo incremento (i) de carga. Nesta etapa, todos os cabos estão em regime elástico e contribuem para a rigidez da estrutura.

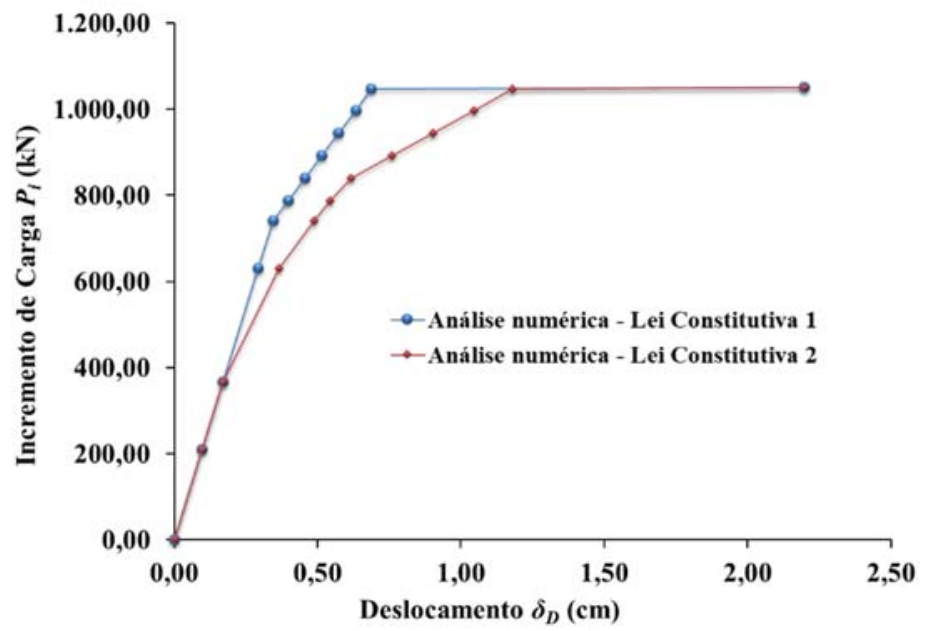

Figura 11 - Curva carga versus deslocamento vertical em $D\left(\delta_{D}\right)$ (lei constitutiva 1 e 2 ) 
A segunda etapa, compreende o trecho entre o terceiro incremento até o quinto incremento. Nesta etapa, todos os cabos estão no trecho elastoplástico e as deformações são maiores para os mesmos incrementos de carga em relação à análise pela lei constitutiva 1 , ou seja, a rigidez do sistema diminui.

$\mathrm{Na}$ terceira etapa, que compreende o trecho entre o sexto incremento até o colapso, o cabo $\overline{\mathrm{BD}}$ escoa e a rigidez do sistema continua diminuindo, já que neste caso, apenas os cabos $\overline{\mathrm{AD}}$ e $\overline{\mathrm{CD}}$ contribuem para a rigidez do sistema. No décimo primeiro incremento os cabos $\overline{\mathrm{AD}}$ e $\overline{\mathrm{CD}}$ também escoam e acontece o colapso da estrutura. Observa-se que, mesmo com o escoamento dos três cabos, há o equilíbrio da estrutura em uma nova posição, com $\delta_{D}=2,199 \mathrm{~cm}$, devido à contribuição da parcela geométrica da rigidez da estrutura na análise.

Da análise conclui-se que, o colapso da estrutura ocorre com a mesma carga limite $P_{u}=1.050,00 \mathrm{kN}$, porém com maiores deslocamentos a cada incremento de carga quando comparada com a análise feita considerando a lei constitutiva 1. Nesta carga de $1.050,00 \mathrm{kN}$ os deslocamentos são os mesmos para as leis contitutiva 1 e 2 . Para a convergência da solução foram necessários no máximo quatro iterações e o tempo de processamento computacional total foi de dois segundos, utilizando o mesmo computador citado anteriormente.

\section{Conclusões}

O objetivo deste trabalho foi apresentar uma formulação teórica, geometricamente exata, para a análise não linear, geométrica e material, de estruturas de cabos de aço suspensos admitindo a sua configuração inicial parabólica, através do método dos elementos finitos, implementando-a em um software para executar a análise tanto elástica quanto elastoplástica dessas estruturas.

$\mathrm{Na}$ formulação do elemento finito, as equações de equilíbrio foram obtidas a partir do princípio dos trabalhos virtuais, considerando-se o equilíbrio do elemento na posição deslocada, tanto na fase elástica quanto na fase elastoplástica. O programa ASTRAS desenvolvido, mostrou-se eficiente na análise dos exemplos apresentados, confirmando a expectativa da potencialidade da formulação adotada.

A adoção da equação da parábola para a definição da configuração inicial de equilíbrio da estrutura, mostrou-se eficiente na geração da malha de elementos finitos e na determinação da geometria final do cabo e das forças envolvidas no problema. Os 
exemplos apresentaram excelente correlação entre os resultados analíticos e numéricos ao se considerar as configurações iniciais das estruturas de cabos como parábola.

Os exemplos apresentados, considerando a análise não linear geométrica e análises não linear material e geométrica, apesar dos pequenos deslocamentos envolvidos nos problemas, permitiram verificar claramente a contribuição da matriz de rigidez geométrica no deslocamento final das estruturas, além de permitir estender de forma mais realista o comportamento do cabo, desde a fase inicial até o colapso, considerando o comportamento elastoplástico perfeito e o comportamento elastoplástico dos cabos. Nos exemplos apresentados, o programa executou entre três e cinco iterações para a resolução dos problemas com vários tipos de malhas para o completo processamento computacional, utilizando-se um computador DELL XPS com processador Intel core i5, CPU de $1,80 \mathrm{GHz}$, memória RAM de $8 \mathrm{~GB}$ e $256 \mathrm{~GB}$ de SSD.

Finalmente, a aplicação do programa ASTRAS na análise das estruturas de cabos suspensos, confirma a consistência e a eficiência da formulação desenvolvida e sua aplicabilidade em casos práticos.

\section{Agradecimentos}

Os autores são gratos à CAPES - Coordenação de Aperfeiçoamento de Pessoal de Nível Superior e ao CNPq - Conselho Nacional de Desenvolvimento Científico e Tecnológico pelo apoio recebido para a realização deste trabalho de pesquisa.

\section{Referências bibliográficas}

Abad, M. S. A.; Shooshtari, A.; Esmaeili, V.; Riabi, A. N. Nonlinear analysis of cable structures under general loadings. Finite Elements in Analysis and Design, v.73, p.11-19, 2013.

Bathe, K. J. Finite element procedures in engineering analysis. New Jersey, Prentice-Hall, Inc., Englewood Cliffs, 1982.

Beer, F. P.; Johnston, E. R; Elliot R. E., 2006. Mecânica Vetorial para Engenheiros - Estática. 7ạed. Rio de Janeiro. McGraw-Hill Interamericana do Brasil.

Costa, R. S., 2014. Formulação para a Análise Avançada de Sistemas Estruturais Formados por Cabos e Treliças Espaciais Visando à Aplicação em Torres Estaiadas para Linhas de Transmissão. Tese de Doutorado. Programa de Pós-Graduação em Engenharia de Estruturas, Escola de Engenharia, Universidade Federal de Minas Gerais, Belo Horizonte.

Hibbeler, R. C., 2011. Estática: Mecânica para Engenharia. 12a ed. São Paulo. Pearson Prentice Hall.

Irvine, H. M., 1975. Statics of suspend cables. Journal of the Engineering Mechanics Division, v. 101, no 3, pp. 187-205.

Irvine, H. M., 1981. Cable structures. Cambridge Ma./London, The MIT Press. 
Kim, S. ; Won, D. H.; Lee, K.; Kang, Y. J. Structural Stability of cable-stayed bridges. International Journal of Steel Structures, v.15(3), p.743-760, 2015.

Lavall, A. C. C., 1996. Uma Formulação Teórica Consistente para a Análise Não linear de Pórticos Planos pelo Método dos Elementos Finitos Considerando Barras com Imperfeições Iniciais e Tensões Residuais nas Seções Transversais. Tese de Doutorado. Escola de Engenharia de São Carlos. Universidade de São Paulo.

Leite, F. N., 2000. Formulação Teórica Consistente para Análise Não Linear de Estruturas Treliçadas Espaciais. Dissertação de Mestrado. Programa de Pós-Graduação em Engenharia de Estruturas, Escola de Engenharia, Universidade Federal de Minas Gerais, Belo Horizonte.

Leonard, J.W., 1988. Tension Structures: behavior and analysis of cable structures. New York. McGraw Hill.

Pai, P. F., Nayfeh, A. H. A new method for the modeling of geometric nonlinearities in structures. Computer and Structures, v. 53, ${ }^{\circ} 4$, p. 877-895, 1994.

Pimenta, P. M., 1989. Derivation of Tangent Stiffness Matrices of Simple Finite Elements - I. Straight Bar Elements. Boletim Técnico do Departamento de Engenharia de Estruturas e Fundações, 8912. São Paulo, EPUSP.

Süssekind, J. C., 1987. Curso de análise estrutural. $8^{\circ}$. ed. Rio de Janeiro: Globo. 